\title{
L'élongase 2 et les acides gras polyinsaturés
}

\section{Acteurs majeurs de l'inflammation}

Ronan Thibaut*, Mathilde Laubert*, Tina Ejlalmanesh, Fawaz Alzaid

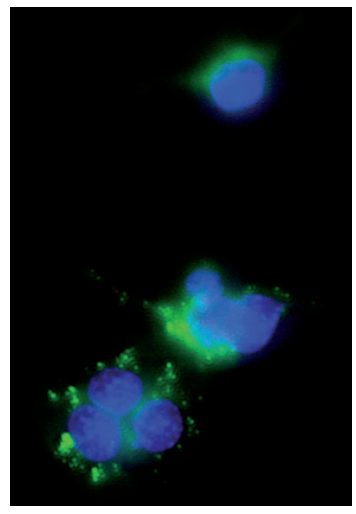

Centre de recherche des Cordeliers, Inserm U1138, IMMEDIAB (Immunity and Metabolism of Diabetes Laboratory), Sorbonne Université, Université de Paris, 15 rue de l'École de Médecine, 75006 Paris, France.

*Co-premier auteurs

fawaz.alzaid@inserm.fr

des membranes plasmiques et intracellulaires [1]. Avec les glucides et les acides aminés, certains acides gras, les triglycérides, permettent, par leur dégradation, la production d'adénosine triphosphate (ATP), une source d'énergie importante pour les cellules et l'organisme [2]. Les lipides qui sont retrouvés dans les cellules peuvent être d'origine endogène ou exogène. De nombreuses cellules sont en effet capables de synthétiser elles-mêmes certains acides gras, dits non essentiels, par la voie de la lipogenèse. Ce processus a lieu dans le cytosol et utilise l'acétyl-coenzyme A (acétyl-CoA) comme précurseur. Si de nombreuses cellules produisent des acides gras par ce processus, seuls les tissus adipeux ont une capacité de stockage de ceux-ci en conditions physiologiques. Ce stockage se fait sous la forme de triglycérides, synthétisés à partir des acides gras. Dans des conditions lipolytiques (comme lors d'une insulino-résistance, par exemple), les adipocytes peuvent libérer des acides gras qui seront transmis à d'autres organes, comme le foie ou les muscles, par la circulation sanguine. Certains acides gras, dits essentiels, comme l'acide linoléique (AL) et l'acide linolénique (ALA), ne peuvent être synthétisés par les cellules : ils sont d'origine alimentaire et permettent de synthétiser les acides gras $\omega$ n-3 et les $\omega n-6$ [3] .

Les lipides extracellulaires, issus de la lipolyse ou de l'alimentation, interagissent avec les cellules selon plusieurs mécanismes : ils peuvent entrer dans la cellule par diffusion passive à travers la membrane plasmique, ou par un transport facilité via des protéines membranaires (comme CD36 ou les FATP, pour fatty acid transporter proteins). Ils peuvent aussi se lier à différents récepteurs, tels que des récepteurs couplés aux protéines $G$ ou les récepteurs de l'immunité innée, les TLR (Toll-like receptor) [4].

Vignette (Photo @ Fawaz Alzaid) 
Après leur entrée dans la cellule, ces acides gras à longue chaîne subissent des modifications structurales. Celles-là interviennent dans le réticulum endoplasmique et sont assurées par deux familles d'enzymes: les désaturases et les élongases (દlovl) [4]. Les désaturases modulent leur taux de saturation, tandis que les élongases modulent la longueur de leurs chaînes carbonées. Chez l'homme et le rat, sept sous-types d'élongases ont été identifiés, clonés et caractérisés (Elovl1-7). Elovl2 et Elovl5 permettent la synthèse endogène d'acides gras à longues chaînes (dont la chaîne carbonée est constituée de plus de 16 carbones). Ces deux enzymes synthétisent, à partir des acides gras provenant de l'alimentation, l'acide docosahexaénoïque (DHA) et l'acide docosapentaénoïque (DPA), précurseurs respectivement des acides gras $\omega n-3$ et $n-6$ (Figure 1 ). Le DHA peut également être synthétisé à partir d'acyl-CoA, et, dans ce cas, de manière endogène [4]. Plusieurs études ont mis en évidence un taux anormalement élevé de DHA endogène en l'absence d'expression d'Elovl2 [5]. Le DHA est un acide gras polyinsaturé à 22 carbones qui est essentiel pour les mammifères. Il est notamment important pour la croissance et lors du développement. Il peut provenir de l'alimentation (via les poissons gras, par exemple) ou être synthétisé de manière endogène, grâce aux élongases. Il constitue une part importante de la membrane plasmique de certaines cellules : il représente, par exemple, environ la moitié des acides gras totaux localisés dans les membranes discales externes des photorécepteurs [6]. Il est également l'acide gras principal contenu dans les spermatozoïdes chez l'homme et une sous-expression, ou une inhibition, de l'expression d'Elvol2, induit des troubles de la synthèse des acides gras à longues chaînes et de la spermatogenèse [7]. Dans les autres tissus, la proportion de DHA dans les membranes est relativement faible, mais elle peut augmenter, par exemple après une supplémentation par un régime alimentaire.

\section{L'expression d'Elovl2 dans les tissus et sa régulation}

Les élongases présentent des caractéristiques qui peuvent être très différentes. Leurs activités peuvent être restreintes à une étape de l'élongation des acides gras. Elles peuvent également être spécifiques de certains organes. Si certaines d'entre elles sont exprimées de façon ubiquitaire, d'autres sont en effet exprimées spécifiquement dans certains tissus (Tableau I) [3]. Certains acides gras à longues chaînes sont ainsi présents dans des proportions distinctes selon les organes en fonction de la présence ou l'absence de ces enzymes. Par exemple, Elovl4, qui permet la synthèse des acides gras saturés et polyinsaturés à très longues chaînes (plus de 28 carbones) est fortement exprimée par les cellules de la rétine.

Le niveau d'expression d'Elovl2 dépend de nombreux facteurs. En particulier, des facteurs génétiques jouent un rôle important. Des polymorphismes de nucléotide unique (SNP) ont été retrouvés sur le gène, dans la région rs953413 localisée au niveau d'un enhancer spécifique des cellules hépatiques. Un des allèles identifiés augmente la fixation des facteurs de transcription HNF4a (hepatocyte nuclear factor-4a) et FOXA (Forkhead box A) à I'ADN de ces cellules, ce qui induit l'expression d'Elovl2 [8]. Ce SNP a été associé à une concentration sérique plus faible de certaines espèces lipidiques, comme l'acide arachidonique et les acides gras polyinsaturés. Ces facteurs génétiques pourraient donc avoir un rôle important sur la fonction d'elovl2 [9]. D'autres SNP retrouvés sur le gène codant Elovl2 n'ont toutefois aucun impact sur les concentrations des différentes espèces lipidiques dans le sang. Seules certaines régions régulatrices du gène ont donc un réel impact fonctionnel sur Elovl2 [10].

Les principaux facteurs cellulaires connus pour réguler l'expression des élongases sont les facteurs de transcription de la famille PPAR (peroxisome proliferator-activated receptor) et la protéine de liaison aux éléments régulateurs des stérols lc (SREBP-lc pour sterol regulatory element-binding transcription factor 1) [11]. En effet, dans des hépatocytes de rat, la surexpression de SREBP-lc entraîne une augmentation d'expression d'Elovl2. En revanche, PPAR-a ne semble pas jouer de rôle important dans l'expression d'Elovl2, même s'il semble indispensable pour la régulation d'Elovl5 et 6 [11]. Dans les larves de poisson-zèbre, un autre récepteur nucléaire, LXR (liver $X$ receptor, aussi appelé récepteur des oxystérols) est également important pour la stimulation de l'expression d'Elovl2 [12].

Des hormones peuvent contribuer à la régulation de la transcription d'Elovl2. Il a été montré que les œstrogènes induisaient la production de la protéine dans des cellules de cancer du sein $[13,14]$ et au niveau de la rétine, mais pas dans le foie. Son expression est aussi modulée selon le rythme circadien [15]. À noter qu'une forte concentration en acides gras saturés dans le sang diminue la transcription d'Elovl2 [14].

Récemment, un lien entre âge et méthylation du promoteur du gène codant عlovl2 a été mis en évidence dans la rétine et le foie, la méthylation augmentant avec l'âge de l'individu [16, 17]. Aucun mécanisme précis expliquant ce phénomène n'a cependant été décrit. II semble néanmoins important puisque la diminution de l'expression d'Elovl2 pourrait accélérer le vieillissement de la rétine.

\section{Elovl2 et les acides gras polyinsaturés $\omega n-3$, des facteurs clés dans la polarisation des macrophages}

Une quantité excessive d'acide gras saturés et polyinsaturés de type $\omega n-6$ a été reliée à différentes maladies, telles que le cancer, les maladies cardio-vasculaires ou les maladies inflammatoires. Les acides gras saturés, comme le palmitate, ont un rôle pro-inflammatoire. Ils induisent la polarisation des macrophages vers le type $\mathrm{Ml}$, pro-inflammatoire, contrairement au type M2 impliqué dans les processus d'angiogenèse, de 
Alimentation

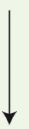

C18:3 (acide linolénique)

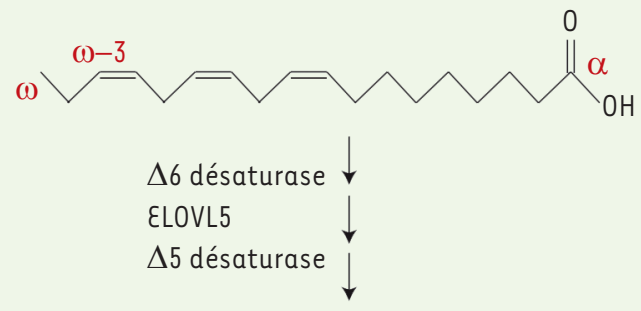

C20:5 (acide éicosapentaénoïque)

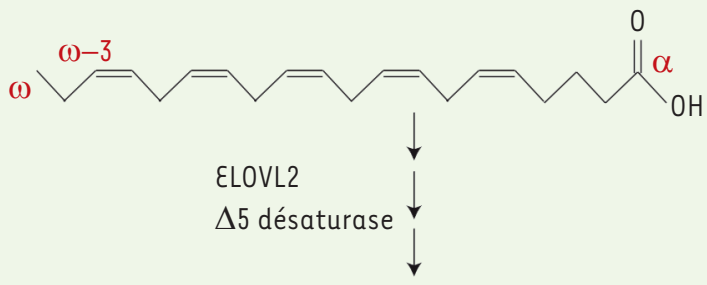

C20:6 (acide docosahexaénoïque)

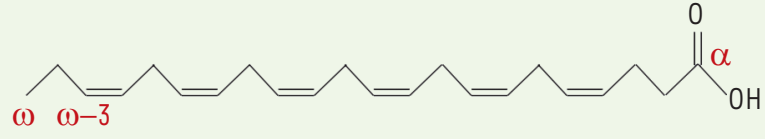

Figure 1. Voie de synthèse simplifiée du DHA à partir de l'acide linolénique provenant de l'alimentation. Cx:y : x est le nombre d'atomes de carbone formant la chaîne d'acides gras et y le nombre d'insaturations (ou de doubles liaisons $C=C$ ). Les enzymes impliquées au cours des différentes étapes sont indiquées. Les atomes de carbone $\alpha, \omega$ et $\omega n-3$, sur lesquels repose la nomenclature des acides gras sont mentionnés en rouge.

réparation ou de remodelage tissulaire. S'il fut d'abord montré que le palmitate activait les macrophages en stimulant la voie impliquant le TLR4 (Toll-like receptor 4), un récepteur de l'immunité innée dédié à la reconnaissance des produits bactériens comme les LPS (lipopolysaccharides), il est maintenant démontré que ces acides gras facilitent, indépendamment du TLR, l'activation de la kinase JNK (c-Jun N-terminal kinase), impliquée dans la polarisation pro-inflammatoire des macrophages [18]. Les acides gras polyinsaturés $\omega n-3$ apparaissent bénéfiques dans un contexte d'inflammation chronique. Le DHA améliore en effet de nombreuses maladies à caractère inflammatoire, telles que l'athérosclérose et le diabète de type 2.

Les lipides sont essentiels à la fonction des cellules. Une étude réalisée sur des cellules de Küpffer, les macrophages spécifiques du foie, a montré l'importance des acides gras polyinsaturés, notamment le DHA et I'EPA, dans l'activation, dans ces cellules, du facteur de transcription PPAR- $\gamma$, essentiel pour leur polarisation vers un phénotype antiinflammatoire [19]. L'activation par le DHA de GPR120, un récepteur couplé aux protéines $G$, conduit à l'inhibition du facteur de trans- cription NF-KB (nuclear factor $-\kappa B$ ) responsable de la transcription de nombreuses cytokines inflammatoires, favorisant ainsi un phénotype anti-inflammatoire de ces cellules [20].

Dans les macrophages, le métabolisme des acides gras polyinsaturés au cours du processus inflammatoire est en fait modulé selon deux phases. Dans des macrophages de souris, la concentration en acides gras polyinsaturés $(\omega n-3$ et $n-6)$ diminue en effet rapidement après activation du TLR4. Cette réduction est corrélée à une diminution de l'activité de gènes qui participent à la synthèse des acides gras polyinsaturés (Fads1 et 2 [fatty acid desaturase 1,2], et Elovl5). Une réponse plus tardive se met ensuite en place, caractérisée par une augmentation de la concentration intracellulaire d'acides gras polyinsaturés, résultant de l'activation de la transcription des gènes responsables de leur synthèse [21].

Une étude, réalisée chez des souris invalidées pour le gène Elovl2, a révélé le rôle anti-inflammatoire essentiel du DHA sur les cellules du tissu adipeux. En effet, en l'absence d'Elovl2, et donc des acides gras que l'enzyme synthétise, une prédominance de macrophages proinflammatoires a été observée. Les acides gras polyinsaturés jouent donc un rôle important non seulement dans la mise en place des processus inflammatoires, dans une première phase, mais également dans leur résolution, lors d'une seconde phase [22].

Ces différentes observations suggèrent donc un rôle possible de l'élongase عlovl2 dans l'adaptation du métabolisme lipidique des macrophages. Néanmoins, les mécanismes utilisés par les cellules myéloïdes afin de moduler leur métabolisme lipidique, notamment via les acides gras polyinsaturés, et donc impliquant l'enzyme Elovl2, qui leur permettent de passer d'un phénotype pro-inflammatoire à un phénotype antiinflammatoire, restent à déterminer.

Au-delà de leur rôle dans la polarisation des macrophages, les acides gras polyinsaturés participent à un ensemble d'actions impliquées dans la résolution des processus inflammatoires.

\section{Elovl2 et acides gras polyinsaturés $\omega$ n-3, des acteurs majeurs de la résolution de l'inflammation}

Les acides gras polyinsaturés $\omega n-3$ occupent une place centrale dans le processus inflammatoire. Ce processus comporte plusieurs phases régulées par des programmes transcriptionnels distincts. Durant une première phase, qui correspond à une inflammation aiguë, les cellules immunitaires, comme les macrophages, produisent des cytokines pro-inflammatoires et des chimiokines 


\begin{tabular}{|c|c|c|}
\hline Élongase & Substrats & $\begin{array}{l}\text { Distribution } \\
\text { tissulaire }\end{array}$ \\
\hline Elovll & $\begin{array}{l}\text { Acides gras saturés C18-C24 } \\
\text { Acides gras monoinsaturés } \\
\text { C18-C24 }\end{array}$ & $\begin{array}{l}\text { Ubiquitaire, forte dans } \\
\text { le cerveau }\end{array}$ \\
\hline Elovl2 & $\begin{array}{l}\text { Acides gras polyinsaturés } \\
\text { C18-C22 }\end{array}$ & $\begin{array}{l}\text { Foie, testicules, glande } \\
\text { parathyroïde, placenta }\end{array}$ \\
\hline Elovl3 & $\begin{array}{l}\text { Acides gras saturés } \mathrm{C} 18-\mathrm{C} 24 \\
\text { Acides gras monoinsaturés } \\
\text { C18:1 }\end{array}$ & $\begin{array}{l}\text { Testicules, peau, faible } \\
\text { expression ubiquitaire }\end{array}$ \\
\hline Elovl4 & $\begin{array}{l}\text { Acides gras saturés et } \\
\text { polyinsaturés } \text { C24 et > C24 }\end{array}$ & $\begin{array}{l}\text { Thymus, peau, } \\
\text { testicules, intestin, } \\
\text { ovaires, prostate }\end{array}$ \\
\hline Elovl5 & $\begin{array}{l}\text { Acides gras polyinsaturés } \\
\text { C18-C22 }\end{array}$ & Ubiquitaire \\
\hline Elovl6 & $\begin{array}{l}\text { Acides gras saturés, } \\
\text { monoinsaturés et } \\
\text { polyinsaturés }\end{array}$ & Ubiquitaire \\
\hline Elovl7 & Acides gras saturés $\mathrm{C} 18$-C22 & $\begin{array}{l}\text { Ubiquitaire (sauf foie et } \\
\text { muscles) }\end{array}$ \\
\hline
\end{tabular}

Tableau I. Substrats et distribution tissulaire des élongases 1-7 chez l'homme. Cx:y : $x$ est le nombre d'atomes de carbone formant la chaîne d'acides gras et y le nombre d'insaturations (ou de doubles liaisons $C=C$ ) (d'après [31]) (human protein atlas: https://www.proteinatlas.org).

qui permettent le recrutement rapide dans le tissu inflammé d'autres cellules immunitaires, telles que les granulocytes neutrophiles. Cette phase est suivie d'une seconde phase de résolution pendant laquelle des molécules anti-inflammatoires produites par les cellules présentes mettent progressivement fin à l'inflammation et permettent la réparation tissulaire [23]. Les acides gras polyinsaturés $\omega n-3$ sont les précurseurs de toute une classe de lipides qui sont impliqués dans la phase de résolution de l'inflammation: les SPM (specialized proresolving mediators). Cette classe de lipides, constituée de plusieurs sous-familles, résolvines, protectines, marésines, est synthétisée par deux types d'enzymes: les cyclooxygénases et les lipoxygénases. L'EPA est précurseur des résolvines de la série $\varepsilon$ et le DHA des résolvines de la série $D$, des protectines et des marésines. Au cours de l'inflammation, un changement s'opère dans les médiateurs lipidiques qui sont produits par les cellules. En effet, au début de l'inflammation, les prostaglandines et leucotriènes, pro-inflammatoires, qui sont synthétisés à partir de l'acide arachidonique, sont majoritaires. Ils sont ensuite progressivement remplacés par les SPM, produits à partir des acides gras polyinsaturés $\omega n-3$ par action de l'acétyl-cyclooxygénase [23]. Cette cinétique se retrouve dans la cellule. En effet, les macrophages stimulés par un agoniste du TLR4 (par exemple le lipopolysaccharide bactérien, à l'origine d'une réponse inflammatoire), réduisent rapidement leur production de lipides anti-inflammatoires. Cette diminution permet le déclenchement d'un programme transcriptionnel pro-inflammatoire. Par la suite, l'activation du facteur de transcription SREBP-1 induit la transcription de gènes impliqués dans la biosynthèse des acides gras, comme Elovl2, ce qui provoque l'accumulation progressive de lipides à action anti-inflammatoire, tels que le DHA ou le DPA, qui vont progressivement éteindre la réponse inflammatoire. Ces changements transcriptionnels, en deux phases successives, permettent ainsi l'initiation, puis la résolution du processus inflammatoire [24]. Des macrophages déficients pour le gène NCoR (nuclear receptor co-repressor) présentent une moindre réponse pro-inflammatoire suite à la stimulation du TLR4. La délétion de NCoR entraîne en effet une augmentation de l'expression des gènes cibles du récepteur nucléaire LXR, dont Elovl5 et de nombreux gènes liés au métabolisme des acides gras. II en résulte une augmentation de la production d'acides gras $\omega n-3$ et de DHA par les cellules, avec une activation réduite de NF-KB et donc une production plus faible de cytokines proinflammatoires, comme I'IL(interleukine)-1 ou le TNF- $\alpha$ (tumor necrosis factor alpha). Bien que son implication spécifique dans ce processus n'ait pas été montrée, il est plus que probable qu'Elvol2, au même titre qu'Elovl5, joue un rôle important dans l'augmentation de la production des acides gras $\omega n-3$ par les cellules n'exprimant pas NCoR [25].

L'action des SPM sur le tissu inflammé implique différentes cellules (Figure 2). Ils limitent en effet l'influx de granulocytes neutrophiles en inhibant leur migration trans-endothéliale, et réduisent leur production d'espèces réactives de l'oxygène. Ils augmentent la capacité des macrophages à phagocyter les pathogènes, mais aussi les cellules en apoptose et les débris cellulaires. Ils ont une action inhibitrice sur les granulocytes éosinophiles, sur les cellules NK (natural killer) ainsi que sur les lymphocytes T. Cette action sur de multiples cellules immunitaires permet un arrêt efficace du processus inflammatoire [23].

\section{Elovl2 et les acides gras polyinsaturés $\omega n-3$ dans l'obésité et le diabète}

Les SPM ont également un rôle dans l'inflammation chronique et les maladies chroniques à composante inflammatoire, comme la pancréatite chronique, l'arthrite ou l'obésité et le diabète de type 2 [24]. Par leur action anti-inflammatoire, les acides gras $\omega n-3$ ont ainsi un rôle important dans l'obésité et le diabète de type 2. En effet, l'inflammation du tissu adipeux est I'un des éléments participant à l'obésité et au développement progressif du diabète de type 2. L'obésité est 
une diminution du taux d'acides gras circulants, réduisent le stockage hépatique de lipides et la sensibilité du foie à l'insuline [26]. Dans le pancréas, l'expression d'Elovl2 et le DHA protègent les cellules $\beta$ pancréatiques de la

associée à une infiltration dans le tissu adipeux et le foie, de diverses cellules immunitaires, notamment de macrophages de type $\mathrm{Ml}$ de phénotype pro-inflammatoire, qui créent une boucle d'auto-amplification de la réponse inflammatoire. Cette infiltration de macrophages proinflammatoires contribue à la mise en place progressive d'une résistance globale à l'insuline et d'un diabète de type 2 [26] et les acides gras polyinsaturés $\omega n-3$ améliorent la sensibilité à l'insuline de souris soumises à un régime très riche en graisse (HFD, high-fat diet).

Comme lors d'une inflammation aiguë, les SPM dérivés des acides gras $\omega n-3$ peuvent aider à résoudre l'inflammation chronique du tissu adipeux. Ils ont une double action : activatrice du facteur PPAR- $\gamma$ et inhibitrice de $N F-\kappa B$, induisant ainsi une polarisation des macrophages vers le type $\mathrm{M} 2$, anti-inflammatoire. Ils diminuent également la production d'eicosanoïdes pro-inflammatoires, à partir de l'acide arachidonique, en réduisant sa proportion dans la membrane plasmique. Les SPM inhibent également la prolifération des adipocytes, limitant ainsi le stockage de lipides dans le tissu adipeux, et favorisent la prédominance des adipocytes de petite taille qui sécrètent moins de cytokines pro-inflammatoires. En activant PPAR- $\gamma$, les acides gras $\omega n-3$ stimulent la production d'adiponectine, une hormone anti-diabétogène qui améliore la sensibilité à l'insuline et augmente la consommation du glucose dans le foie et le muscle squelettique [26]. Ils inhibent aussi la production hépatique de triglycérides. En particulier, l'EPA, qui active PPAR- $\alpha$ et I'AMP kinase (AMPK), stimule la $\beta$-oxydation des acides gras dans le foie, mais aussi, avec le DHA, dans le muscle squelettique. En inhibant les facteurs de transcription SREBP-lc et ChREBP (carbohydrate-responsive element-binding protein), il réduit la lipogenèse hépatique. Combinés, ces différents effets permettent mort induite par glucolipotoxicité, et stimulent la sécrétion d'insuline [27, 28], favorisant ainsi l'action régulatrice de l'insuline sur le métabolisme glucidique et lipidique.

\section{Conclusions et perspectives}

Les acides gras jouent un rôle fondamental dans la physiologie de l'organisme. Ils ont des fonctions capitales dans les cellules: par leur présence dans les membranes, leurs rôles dans la signalisation cellulaire, mais aussi par leur importante participation, du fait de leur catabolisme, à la production d'ATP. Leur variété structurale repose entre autres sur leur taux de saturation et sur la longueur de leur chaîne carbonée, qui sont contrôlés par deux classes d'enzyme: les désaturases et les élongases. L'une de ces élongases, Elovl2, est responsable de la synthèse des acides gras polyinsaturés à longue chaîne, notamment les acides gras polyinsaturés $\omega n$-3. Ces acides gras jouent un rôle prépondérant dans le contrôle des processus inflammatoires. Dans l'obésité et le diabète, ils ont un rôle bénéfique de protection en limitant le stockage de lipides et l'inflammation chronique du tissu adipeux, ainsi qu'en améliorant la sensibilité à l'insuline.

De nombreuses questions restent toutefois sans réponse. Plusieurs études montrant les effets bénéfiques des acides gras à longue chaîne $\omega n-3$ reposent 
sur des apports en acides gras $\omega \mathrm{n}-3$ exogènes. L'ampleur de la synthèse endogène de ces acides gras, son origine cellulaire, sa régulation et son importance fonctionnelle restent largement inconnues. Le lien qui les lie à l'expression d'Elovl2 et d'autres enzymes impliquées dans leur biosynthèse reste également mal compris. La participation des SPM a également été montrée dans de nombreuses maladies infectieuses mais aussi auto-immunes. Elovl2 et les autres enzymes responsables de la synthèse des SPM semblent donc avoir un rôle potentiellement fondamental dans la régulation des processus inflammatoires pathologiques. Les SPM pourraient ainsi représenter des candidats intéressants pour remédier à de nombreuses maladies présentant une composante inflammatoire.

Les acides gras polyinsaturés pourraient toutefois être néfastes dans certaines maladies. L'expression d'Elovl2 est en effet associée à une diminution de survie des patients atteints de glioblastome. Elovl2, qui permet le maintien de la signalisation par le récepteur de l'EGF (epidermal growth factor), est en effet nécessaire à la survie des cellules souches cancéreuses qui favorisent la progression de la maladie [30]. Un effet pro-tumoral indirect par l'inhibition de la réponse inflammatoire, est également envisageable. $\diamond$

\section{SUMMARY}

Elongase 2 and polyunsaturated fatty acids: Key players in inflammation and type 2 diabetes

Elongases (Elovl) are enzymes which allow elongation of fatty acid chains. They are genetically highly conserved across a number of species among which rat, mice and men. There are seven known elongases ( $\varepsilon$ lovll to 7). These enzymes are implicated in several metabolic diseases. Recent publications have emphasized the importance of Elovl2 in proper cellular and tissue functions. It has many roles in various physiological processes due to its function in $\omega n-3$ and n- 6 fatty acids synthesis. $\omega n-3$ and $n-6$ fatty acids have a role in development and function of the brain and other organs. They also have a central role in inflammatory processes by acting on macrophages which are key cellular players of the innate immune system. As a consequence, these lipid mediators have a paramount importance in diseases at least partially driven by inflammation. The example of obesity and type 2 diabetes highlights the diversity of their cellular and molecular targets and effects. $\diamond$

\section{LIENS D'INTÉRÊT}

Les auteurs déclarent n'avoir aucun lien d'intérêt concernant les données publiées dans cet article.

\section{RÉFÉRENCES}

1. Carvalho CCCR de, Caramujo MJ. The various roles of fatty acids. Molecules $2018 ; 23$.

2. Bonora M, Patergnani S, Rimessi A, et al. ATP synthesis and storage. Purinergic Signal $2012 ; 8$ : 343-57.

3. Kihara A. Very long-chain fatty acids: elongation, physiology and related disorders. J Biochem $2012 ; 152$ : 387-95.

4. Doege $H$, Stahl A. Protein-mediated fatty acid uptake: novel insights from in vivo models. Physiology (Bethesda) $2006 ; 21: 259-68$.

5. Pauter AM, Olsson P, Asadi A, et al. Elovl2 ablation demonstrates that systemic DHA is endogenously produced and is essential for lipid homeostasis in mice. J Lipid Res 2014 ; 55 : 718-28.

6. Stillwell W, Wassall SR. Docosahexaenoic acid: membrane properties of a unique fatty acid. Chem Phys Lipids 2003 ; 126 : 1-27.
7. Zadravec D, Tvrdik P, Guillou H, et al. ELOVL2 controls the level of $n-628: 5$ and $30: 5$ fatty acids in testis, a prerequisite for male fertility and sperm maturation in mice. J Lipid Res $2011 ; 52: 245-55$.

8. Pan G, Cavalli M, Carlsson B, et al. rs 953413 Regulates polyunsaturated fatty acid metabolism by modulating ELOVL2 expression. iScience $2020 ; 23: 100808$

9. Fujii TM de M, Norde MM, Fisberg RM, et al. FADS1 and ELOVL2 polymorphisms reveal associations for differences in lipid metabolism in a cross-sectional population-based survey of Brazilian men and women. Nutr Res $2020 ; 78: 42-9$.

10. Barman M, Nilsson S, Torinsson Naluai $\AA$, et al. Single nucleotide polymorphisms in the fads gene cluster but not the ELOVL2 gene are associated with serum polyunsaturated fatty acid composition and development of allergy (in a Swedish birth cohort). Nutrients $2015 ; 7$ : 10100-15.

11. Wang $\mathrm{Y}$, Botolin D, Xu J, et al. Regulation of hepatic fatty acid elongase and desaturase expression in diabetes and obesity. J Lipid Res 2006 ; 47 : 2028-41.

12. Pinto CL, Kalasekar SM, McCollum CW, et al. Lxr regulates lipid metabolic and visual perception pathways during zebrafish development. Mol Cell Endocrinol $2016 ; 419: 29-43$.

13. González-Bengtsson A, Asadi A, Gao H, et al. Estrogen enhances the expression of the polyunsaturated fatty acid elongase Elovl2 via $\varepsilon R \alpha$ in breast cancer cells. PLoS One 2016; 11 : e0164241.

14. Kim D, Choi JE, Park Y. Low-linoleic acid diet and oestrogen enhance the conversion of $\alpha$-linolenic acid into DHA through modification of conversion enzymes and transcription factors. Br J Nutr 2019; 121 : 137-45.

15. Wang Q, Tikhonenko M, Bozack SN, et al. Changes in the daily rhythm of lipid metabolism in the diabetic retina. PLoS One $2014 ; 9$ : e95028.

16. Taniguchi I, Iwaya C, Ohnaka K, et al. Genome-wide DNA methylation analysis reveals hypomethylation in the low- $\mathrm{CpG}$ promoter regions in lymphoblastoid cell lines. Hum Genomics $2017 ; 11: 8$.

17. Chen D, Chao DL, Rocha L, et al. The lipid elongation enzyme ELOVL2 is a molecular regulator of aging in the retina. Aging Cell $2020 ; 19$ : e13100.

18. Lancaster GI, Langley KG, Berglund NA, et al. Evidence that TLR4 is not a receptor for saturated fatty acids but mediates lipid-induced inflammation by reprogramming macrophage metabolism. Cell Metab 2018 ; 27 : 1096-110.e5.

19. Luo W, Xu $Q$, Wang $Q$, et al. Effect of modulation of PPAR- $\gamma$ activity on Kupffer cells M1/M2 polarization in the development of non-alcoholic fatty liver disease. Sci Rep $2017 ; 7: 44612$.

20. Oh DY, Talukdar S, Bae EJ, et al. GPR120 is an omega-3 fatty acid receptor mediating potent anti-inflammatory and insulin-sensitizing effects. Cell $2010 ; 142: 687-98$.

21. Ménégaut $L$, Jalil A, Thomas $C$, et al. Macrophage fatty acid metabolism and atherosclerosis: The rise of PUFAs. Atherosclerosis $2019 ; 291: 52-61$.

22. Talamonti $\varepsilon$, Pauter AM, Asadi A, et al. Impairment of systemic DHA synthesis affects macrophage plasticity and polarization: implications for DHA supplementation during inflammation. Cell Mol Life Sci 2017 ; 74 : 2815-26.

23. Basil MC, Levy BD. Specialized pro-resolving mediators: endogenous regulators of infection and inflammation. Nat Rev Immunol 2016; 16 : 51-67.

24. Oishi Y, Spann NJ, Link VM, et al. SREBPI contributes to resolution of proinflammatory tlr 4 signaling by reprogramming fatty acid metabolism. Cell Metab $2017 ; 25: 412-27$.

25. Li P, Spann NJ, Kaikkonen MU, et al. NCoR repression of LXRs restricts macrophage biosynthesis of insulin-sensitizing omega 3 fatty acids. Cell $2013 ; 155: 200-14$.

26. Wang Y, Huang F. N-3 Polyunsaturated fatty acids and inflammation in obesity: local effect and systemic benefit. Biomed Res Int $2015 ; 2015$ : 581469.

27. Kalupahana NS, Claycombe KJ, Moustaid-Moussa N. (n-3) Fatty acids alleviate adipose tissue inflammation and insulin resistance: mechanistic insights. Adv Nutr 2011 ; 2 : 304-16.

28. Cruciani-Guglielmacci C, Bellini L, Denom J, et al. Molecular phenotyping of multiple mouse strains under metabolic challenge uncovers a role for Elovl2 in glucose-induced insulin secretion. Mol Metab $2017 ; 6: 340-51$.

29. Bellini L, Campana M, Rouch C, et al. Protective role of the ELOVL2/ docosahexaenoic acid axis in glucolipotoxicity-induced apoptosis in rodent beta cells and human islets. Diabetologia $2018 ; 61: 1780-93$.

30. Gimple RC, Kidwell RL, Kim LJy, et al. Glioma stem cell-specific superenhancer promotes polyunsaturated fatty-acid synthesis to support EGFR signaling. Cancer Discov 2019 ; 9 : 1248-67.

31. Ohno Y, Suto S, Yamanaka M, et al. ELOVL1 production of C24 acyl-CoAs is linked to C24 sphingolipid synthesis. Proc Natl Acad Sci USA 2010 ; 107 : 18439-44.

TIRÉS À PART

F. Alzaid 